\title{
Paraneoplastic Acral Vascular Syndrome Due to Pulmonary Adenocarcinoma: Case Report
}

\author{
Jaouad Yousfi $^{1 *}$, Soukaina Oumli1 ${ }^{1}$, Mouna Zahlane ${ }^{1}$, Lamiaa Essaadouni ${ }^{1}$
}

${ }^{1}$ Service de Medicine Interne, Hôpital Arrazi, CHU Mohamed VI, Marrakech, Maroc

DOI: $10.36347 /$ sjmcr.2021.v09i03.012

| Received: 16.02.2021 | Accepted: 27.02.2021 | Published: 15.03.2021

*Corresponding author: Jaouad Yousfi

Abstract

Case Report

Acral vascular syndrome is the translation of a general or locoregional pathology. It is associated with atherosclerotic, thromboembolic, or connective tissue diseases. Less often, it can be related to malignancy. However, the etiological assessmentshould not delay the treatment in order to preserve functional prognosis. We present the case of a patient with paraneoplastic acral vascular syndrome as an initial manifestation of lung cancer. In the absence of an identifiable iatrogenic cause, occupational diseases, atherosclerosis or systemic disease, digital necrosis should alert the examiner to search for a neoplastic etiology namely a solid cancer or haemopathy.

Keywords: Acral vascular syndrome, paraneoplastic, lung cancer.

Copyright $(\mathcal{O} 2021$ The Author(s): This is an open-access article distributed under the terms of the Creative Commons Attribution 4.0 International License (CC BY-NC 4.0) which permits unrestricted use, distribution, and reproduction in any medium for non-commercial use provided the original author and source are credited.

\section{INTRODUCTION}

Les syndromes paranéoplasiques représentent l'ensemble de signes cliniques et, ou biologiques survenant au cours d'un processus néoplasique, indépendamment de tout envahissement tumoral ou localisation secondaire. L'association de troubles trophiques digitaux et cancer reste exceptionnelle. Nous rapportons le cas d'un patient avec une ischémie digitale ayant révélé un adénocarcinome pulmonaire.

\section{ObServation}

Nous rapportons le cas d'un patient de 56 ans, non tabagique, ayant développé, trois semaines avant son hospitalisation, une thrombose veineuse profonde du membre inferieur droit et qui se présentait pour une cyanose intéressant les 2 mains plus marquée à droite (figures 1,2) évoluant dans un contexte d'apyrexie et d'altération progressive de l'état général. L'examen clinique avait trouvé un patient conscient apyrétique, eupnéique. Une cyanose froide des doigts sans nécrose était objectivée. L'examen abdominal retrouvait une masse abdominale paramédiane droite de $4 \mathrm{~cm}$ de grand axe, fixe adhérente au plan profond.

L'echodoppler artériel des deux membres supérieurs n'avait pas trouvé d'anomalies morphologiques ni dynamiques. Le scanner abdominal, avait objectivé une masse pariétale médiane infiltrant le muscle droit, latéralisée à droite de $65 \mathrm{mmx} 49 \mathrm{~mm}$ étendue sur 58mm, associée à des nodules de péritonéaux d'allure secondaires. Le bilan biologique comportant, l'hémogramme, la fonction rénale, le bilan hépatique et lipidique était normal, tandis que la vitesse de sédimentation était accélérée à $69 \mathrm{~mm}$, CRP augmentée à $182.6 \mathrm{mg} / \mathrm{l}$. Le dosage des marqueurs tumoraux avait montré une forte augmentation de CA125 à 2823(0.0-27), CA15.3 à 182.3 (0.0-25), alors que l'AFP, CEA, et le CA19.9 étaient normaux. L'étude histologique de la masse abdominale était en rapport avec une localisation métastatique d'un adénocarcinome, un complément d'étude par une immunohistochimie était en faveur d'un adénocarcinome pulmonaire, mais on avait pas objectivé de lésions radiologiques sur la radiographie des poumons ni à la TDM thoracique, ainsi que l'état général altéré du patient n'avait pas permis de compléter l'exploration pulmonaire par une bronchoscopie diagnostique.

Deux semaines plus tard, le patient avait présenté une dyspnée aigue revalant une embolie pulmonaire massive ayant nécessité une prise en charge en réanimation pendant 05jours.

L’évolution de lésions cutanées était marquée par la survenue des lésions ischémiques avec l'installation de nécrose sèche hyperalgique peu contrôlée sous morphinique à libération prolongée. Apres 6 semaines d'hospitalisation, le patient était 
décédé avant de commencer les séances de chimiothérapie.
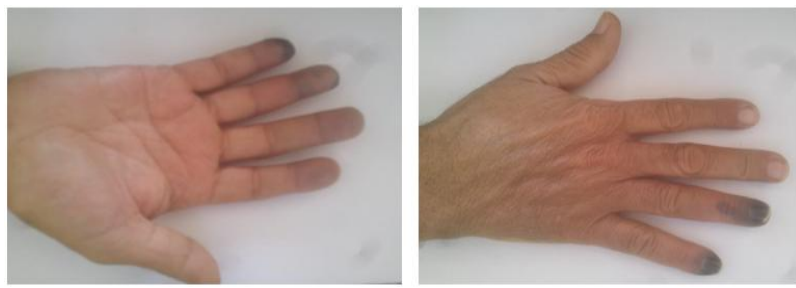

Fig-1-2: Ischémie digitale de la main droite

\section{DISCUSSION}

Les causes des acrosyndromes pouvant évoluer vers une nécrose sont dominées par les connectivites, surtout la sclérodermie systémique, puis les causes professionnelles (microtraumatismes répétés), et plus rarement une vascularite, une cryoglobulinemie, ou un syndrome myéloproliferatif, notamment la thrombocytémie essentielle, et exceptionnellement, les tumeurs solides [1, 2]. Les néoplasies les plus fréquemment en cause sont les carcinomes digestifs, le cancer bronchique et les hémopathies (leucémie, lymphome, myélome et macroglobulinémie de Waldenström) [3].

Ces troubles trophiques peuvent survenir au cours de l'évolution du cancer ou être révélateurs. Dans l'étude de Poszepcszynska-Guigné et al. les acrosyndromes vasculaires précédaient le diagnostic de cancer dans 43,75\% des cas, ce dernier était au stade métastatique dans 41,2\% des cas [4]. Dans notre cas la nécrose digitale a précédé la découverte d'adénocarcinome pulmonaire de quatre semaines.

La physiopathologie des nécroses digitales paranéoplasiques est complexe, souvent multifactorielle. Plusieurs hypothèses ont été évoquées pour expliquer le vasospasme et/ou l'obstruction artérielle : envahissement des nerfs sympathiques, vascularite, hyperviscosité, phénomènes immunologiques, cryoglobulinémie. Les états tumoraux exposent en effet à des thrombophilies acquises d'expression artérielle et acrale [5]. L'hypercoagulabilité au cours du cancer est reconnue comme un facteur thrombogène majeur, induisant le plus souvent des manifestations thrombotiques veineuses ou artérielles. L'hypercoagulabilité peut également être favorisée au cours du cancer par l'existence d'une hyperviscosité liée à un syndrome inflammatoire majeur, une gammapathie monoclonale, une thrombocytose, une cryoglobulinémie, et par la présence des agglutinines froides [6].

La diversité étiologique des nécroses digitales a conduit certains auteurs à adopter une démarche diagnostique. Un interrogatoire et un examen clinique attentif et méthodique permettent dans la grande majorité des cas d'approcher ce diagnostic étiologique, d'orienter les explorations paracliniques [1]. Dans tous les cas, l'enquête étiologique ne devra pas retarder la prise en charge thérapeutique afin de préserverau maximum le pronostic fonctionnel [1, 7].

Le caractère paranéoplasique des nécroses ischémiques digitales est difficile à mettre en évidence, ces patients ont le plus souvent des comorbidités, particulièrement le tabagisme qui favorise également les ischémies digitales. Cependant, la chronologie de ces lésions nécrotiques, qui apparaissent au moment du diagnostic du cancer, évoluent en parallèle et peuvent parfois régresser après sa guérison, laisse supposer une implication directe du cancer dans la physiopathologie comme tout syndrome paranéoplasique [8].

\section{Conclusion}

Les étiologies des acrosyndromes sont nombreuses, dominées par les connectivites chez la femme et les artériopathies chez l'homme. La démarche diagnostique devrait être basé initialement sur une anamnèse et un examen clinique afin d'éliminer une origine néoplasique pouvant engager le pronostic vital.

\section{REFERENCES}

1. Hachulla E, Hatron P-Y. Nécroses digitales. Revue du Rhumatisme monographies. 2012;79(2):96- 100.

2. Langeron P, Mikati A, Redgosz R. Diversité étiologique des artérites digitales. Phlébologie. 1987;40:11 - 20

3. García-Sanz R, Montoto S, Torrequebrada A, De Coca AG, Petit J, Sureda A Waldenströmmacroglobulinaemia: presenting features and outcome in a series with 217 cases. British journal of haematology. 2001;115(3):575- 82.

4. Poszepczynska-Guigné E, Viguier M, Chosidow O, Orcel B, Emmerich J, Dubertret L. Paraneoplasticacral vascular syndrome: epidemiologic features, clinical manifestations, and disease sequelae. Journal of the American Academy of Dermatology. 2002;47(1):47- 52.

5. Stéphan D, Wahl D, Michon-Pasturel U, Kownator S. Les ischémies digitales: diagnostic clinique, paraclinique et étiologique. Journal des Maladies Vasculaires. 2010;35(2):75.

6. Poiraud C, Durant C, Saint-Jean M, Connault J, Barbarot $\mathrm{S}$. Une cause rare de nécroses digitales: la maladie de Hodgkin. La Presse médicale (1983). 2011;40(4):432-5.

7. Abdallah M, Hamzaoui S, Larbi T, Bouslama K, Harmel A, Ennafaa M, Bahloul Z, Rokbani L, Othmani S, Dridi MB, M'rad S. Profil étiologique des nécroses digitales des membres supérieurs: analyse de 25 observations. Journal des maladies vasculaires. 2010 Feb 1;35(1):12-6.

8. Bouchentouf R, Benjelloun A, Aitbenasser MA Ulcérations digitales révélant un carcinome bronchique. Revue de Pneumologie clinique. 2012;68(6):367 - 9. 